\title{
Utilization of Health Care Services and Satisfaction with Care in Adults Affected by Disorders of Sex Development (DSD)
}

\author{
Ute Thyen, $M D^{7}$, Anke Lux, MS², Martina Jürgensen, $P h D^{3}$, Olaf Hiort, $M D^{4}$, and Birgit Köhler, MD, \\ $\mathrm{PhD}^{5}$ \\ ${ }^{1}$ Hospital for Children and Adolescents, University Lübeck, Lübeck, Germany; ${ }^{2}$ Institute for Biometrics and Medical Informatics, \\ University Magdeburg, Magdeburg, Germany; ${ }^{3}$ Institute for Social Medicine and Epidemiology, University Lübeck, Lübeck, Germany; \\ ${ }^{4}$ Hospital for Children and Adolescents, Division of Pediatric and Experimental Endocrinology, University Lübeck, Lübeck, Germany; \\ ${ }^{5}$ Division of Experimental Endocrinology, Charité, Berlin, Germany.
}

BACKGROUND: Disorders of sex development (DSD) are a heterogeneous group of rare genetic disorders of sex determination or differentiation. Evidencebased guidelines concerning gender assignment and surgical and hormonal treatment are limited for many DSD entities, and health care is highly fragmented across various sub-specialties and settings. A lack of informed consent, secrecy about the condition, shame, and impaired sexual and psychosocial functioning may affect satisfaction with care.

OBJECTIVES: The main goal of this study was to describe satisfaction with care in individuals with DSD and to identify factors associated with low satisfaction with care.

METHODS / MAIN MEASURES: Using both biological (chromosomes) and social categories (sex of rearing), we classified participants according to the nomenclature of the European Society for Pediatric Endocrinology/Lawson Wilkins Pediatric Endocrine Society (ESPE/LWPES) consensus statement. We used standardized measures to assess satisfaction with care (CSQ-8), health-related quality of life (SF36), psychological symptoms (BSI), and gender identity (FGI), in addition to self-constructed questionnaires probing experiences with health care and access to self-help groups.

PARTICIPANTS: A total of 110 adults were recruited between January 2005 and December 2007 in four study centers in Germany, Austria, and German-speaking Switzerland.

RESULTS: Reports of half the participants scored below the cut-off indicating low quality of care. Women with XX DSD conditions and virilization (i.e., congenital adrenal hyperplasia) reported the highest scores for satisfaction with care, and women with XY DSD conditions and complete lack of androgen effects reported the lowest scores. Satisfaction with care was positively associated with indicators of psychological well-being.

CONCLUSIONS: Satisfaction with care is lowest among participants with the rarest conditions, highlighting the lack of evidence-based recommendations and the lack of coordination of care. Associations of satisfaction and well-being indicate the need to ensure access to mental health services.

Published online July 17, 2014
KEY WORDS: health services; satisfaction with care; disorders of sex development; adults.

\author{
Abbreviations \\ $\mathrm{CAH} \quad$ Congenital adrenal hyperplasia \\ CAIS Complete androgen insensitivity syndrome \\ CSQ Client satisfaction questionnaire \\ DSD Disorder of sex development \\ DSD-XX-P-F Females with 46, XX DSD and partial andro- \\ gen effects \\ DSD-XY-P-F Females with 46, XY DSD and partial andro- \\ gen effects \\ DSD-XY-C-F Females with 46, XY DSD and complete lack of \\ androgen effects \\ DSD-XY-P-M Males with 46, XY DSD and partial androgen \\ effects \\ PAIS Partial androgen insensitivity syndrome \\ J Gen Intern Med 29(Suppl 3):S752-9 \\ DOI: $10.1007 / \mathrm{s} 11606-014-2917-7$ \\ (c) Society of General Internal Medicine 2014
}

\section{INTRODUCTION}

Disorders of sex development (DSD) are rare diseases, with incidence varying between $<1$ and 20 per 100,000 . They encompass a heterogeneous group of congenital conditions affecting sex determination or differentiation with diverse genetic etiology and varying levels of prenatal androgen effects and different genitalia phenotypes. Earlier nomenclature such as "hermaphroditism," "testicular feminization," or "intersexuality" have been abandoned in favor of a non-stigmatizing nomenclature based on chromosomal sex. ${ }^{1}$ This classification allows the distinction of conditions with 46, XY karyotype and varying degrees of undervirilization, which may be complete (resulting in female gender) or partial (individuals may live in either female or male gender), and conditions with 46, XX karyotype and excess virilization (whereby the vast majority of individuals live as females). A more generic definition of DSD allows us to consider individuals with syndromes or disorders of 
unknown etiology and to increase the sample size of subgroups.

The vast majority of conditions with 46, XX karyotype and varying degrees of atypical virilization are attributable to congenital adrenal hyperplasia $(\mathrm{CAH})$ due to 21hydroxylase deficiency. Since the middle of the 20th century, this has been a well-understood disorder of steroid metabolism, and medical treatment is fairly standardized, with guidelines based on clinical experience. ${ }^{2,3}$ The condition may be detected by metabolic screening of newborns or by identifying virilized genitalia in newborn girls; diagnostic information and counseling becomes available within days. On the contrary, XY-DSD conditions are not always evident at birth, but sometimes manifest only later in childhood or even in adolescence. A multitude of pathogenetic mechanisms including sex determination, differentiation, androgen receptor functioning, and steroid biosynthesis make diagnostic pathways complex; in many conditions the etiology remains undetermined. ${ }^{4}$ Given the rarity of the conditions, the heterogeneity of the clinical symptoms, and variation in both surgical and medical treatments, evidence-based guidelines for XX-DSD but especially for XY-DSD conditions must be based largely on expert recommendations.

In addition to typical challenges of studying rare conditions, abnormalities affecting the genital organs and psychosexual development further increase the difficulties in clinical care for this group of people. ${ }^{5}$ In the past, the potentially stigmatizing effect of the conditions has led to the assumption that physicians may voluntarily withhold information from the patient that they perceived as threatening the patient's personal integrity.

Since the 1960s policies have attempted to help affected individuals by "normalizing" their physical appearance to minimize the effects of being different and thus to reduce stigmatization. This approach became known as the "optimal gender policy," as developed by John Money and colleagues at Johns Hopkins Hospital. ${ }^{6,7}$ Later reports from both clinicians and patient advocacy groups challenged the assumptions that early constructive surgery is in the best interest of the affected individuals. ${ }^{8-10}$ New knowledge became available regarding the effect of prenatal and postnatal androgens on both the development of the sexual organs and the brain, affecting later development of gender identity. ${ }^{11}$ Recent recommendations suggested postponing surgical operations until the children are able to give their consent. The new paradigm became known as the "full consent policy." 12

Adults between 18 and 60 years of age may have experienced wide variation of treatments and services. Patient-reported outcomes such as satisfaction with care may serve as a proxy for the processes associated with quality health care. ${ }^{13}$ For this study, we wished to (1) describe satisfaction with care in adults with various DSD conditions; (2) identify factors associated with low satisfaction with care (type of condition, age group, access to specialist care, membership in patient organizations, disclosure and communication with physicians, satisfaction with surgical outcomes and body functioning, psychological symptoms, and health-related quality of life); and (3) investigate whether access to psychosocial care reduces the burden of psychological symptoms.

\section{METHODS}

This study was part of the clinical evaluation study of the German Network of DSD. The aim of the study was the evaluation of health-related quality of life, psychosexual development, satisfaction with care, coping, and problems associated with diagnoses and therapies in individuals with DSD in all age groups. This article focuses on the findings concerning utilization of health care services, satisfaction with care, unmet needs in adults with DSD, and factors influencing these outcomes.

Study participants were recruited from four study centers in Germany, two in Austria, and two in German-speaking Switzerland between January 2005 and December 2007. Inclusion criteria followed a generic definition of DSD as a discrepancy of chromosomal, gonadal, and phenotypical sex. Included were individuals with a confirmed diagnosis of DSD, as well as individuals with a clinical diagnosis by a physician without laboratory or molecular genetic confirmation. Individuals with Klinefelter or Ullrich-Turner Syndrome, severe psychiatric co-morbidity, or mental disabilities were not included.

Study centers approached their patients attending specialty clinics. Additionally, medical cooperation partners and self-help groups in Germany encouraged their patients/ members to join the study. Every participant received informed consent sheets, including information about data security and a waiver of consent to allow transmission of confidential medical information from the physician identified as the main case manager. The study was approved by the medical ethics committee at the University of Lübeck and ethics review boards at the other study centers.

We assigned the participants to one of four categories according to the nomenclature of the Chicago consensus statement; the classifications "female" and "male" refer to the social gender that the individual lived in and is reported in the study:

1) DSD-XX-P-F: Females with 46, XX DSD and partial androgen effects, predominantly $\mathrm{CAH}$, and few with partial gonadal dysgenesis, aromatase deficiency, and ovotesticular DSD with XX karyotype;

2) DSD-XY-P-F: Females with 46, XY DSD and partial androgen effects included gonadal dysgenesis (partial 
or mixed), disorder of androgen biosynthesis, partial androgen insensitivity syndrome (pAIS), severe hypospadias, cloacal exstrophy, penis agenesis, or unclassified clinical diagnosis of DSD;

3) DSD-XY-C-F: Females with 46, XY DSD and complete lack of androgen effects included complete androgen-insensitivity syndrome (cAIS) and complete gonadal dysgenesis; or

4) DSD-XY-P-M: Males with 46, XY DSD and partial androgen effects included gonadal dysgenesis (partial or mixed), disorder of androgen biosynthesis, micropenis, severe hypospadias, epispadias, XX-male with SRY-translocation, and ovotesticular DSD with $\mathrm{XY}$ karyotype, or unclassified clinical diagnosis of DSD.

\section{MEASURES}

The design and the measures are described in the open-access publication by the authors $\left[{ }^{14}\right.$; http://www.biomedcentral.com/ 1471-2458/9/110]. All interviews related to patient-reported outcomes were conducted by trained psychologists at one of the study centers or at the participant's home, depending on the preference of the participant. We used standardized instruments to assess general outcomes, such as satisfaction with care, quality of life, and psychological outcomes. There are few condition-specific instruments for DSD. We selected questionnaires for psychosexual outcomes after consulting experts in the field and extensive review of the literature. For example, to assess gender dysphoria, we used an instrument developed to measure gender identity disorder. A selfconstructed disease-specific DSD-questionnaire covering specific aspects concerning DSD has been developed in cooperation with support groups and parent organizations (focus group discussions) and academic and medical experts.

Satisfaction with Care. We used the German version of the CSQ-8, ${ }^{15}$ which is a one-dimensional instrument for the assessment of global patient satisfaction. The instrument consists of eight items with four response options on a Likert scale, ranging from low satisfaction or low agreement with a statement to high satisfaction and agreement. The lowest possible score is 8 , and the highest score 32. The authors report excellent reliability and internal consistency ranging from 0.83 to 0.93 .

Health-Related Quality of Life. To assess health-related quality of life, we used the German version of the SF-36, ${ }^{16}$ a well-evaluated standardized instrument including eight subscales (physical function, social function, role physical, role emotional, bodily pain, vitality, mental health, and general health) and two total scales (physical and mental health) with varying response categories (Cronbach's alpha $>0.85)$. Higher scores represent better subjective health.

Psychological Symptoms. Mental health was assessed using the German version of the Brief Symptom Inventory (BSI). ${ }^{17}$ This instrument includes 53 items in nine subscales (somatization, obsessive-compulsive, interpersonal sensitivity, depression, anxiety, hostility, phobic anxiety, paranoid ideation, and psychoticism) and three global characteristic values (global severity, positive symptom distress, positive symptom total). Participants rate the extent to which they have been bothered $(0=$ "not at all" to $4=$ "extremely") in the past week by various symptoms; higher scores indicate a higher level of psychological distress.

Gender Identity. Gender identity was assessed by using the Questionnaire of Gender Identity (FGI). ${ }^{18}$ The 22item questionnaire includes four subscales (feminine gender identity, masculine gender identity, transgender scale, and scale on certainty of belonging to one specific gender). In this analysis, we used the transgender scale; higher scores indicate more gender dysphoria. The lowest possible score is 1, and the highest possible score is 5 .

To assess more general and DSD-specific information, we developed a DSD-Questionnaire for adults including questions about how long it took to establish a diagnosis and whether they: (1) understood the explanations about the diagnosis, (2) had received psychological counseling, (3) had access to specialist care, and (4) were members of a patient organization.

Questions about surgical outcomes asked respondents to rate satisfaction with overall appearance, urological functioning, and sexual functioning in four response categories. Higher scores indicate more satisfaction. We used these items to explain variation in satisfaction with care.

\section{STATISTICAL ANALYSIS}

Data were analyzed using IBM SPSS Statistics software, Version 21. The outcome variable of satisfaction with care is expressed as the mean and standard deviation in the whole group and in the different subgroups. For the comparison of satisfaction regarding the items for diagnosis, receipt of services, surgical outcomes t-tests for independent samples, or methods of analysis of variance (followed by Scheffe's test or Tamhane's test as 
post hoc tests for multiple comparisons) were used. Relationships with scales of standardized instruments (SF-36, BSI, and FGI) were examined using correlation analyses ( $\mathrm{r}=$ Spearman Rank Test). In all tests, $\mathrm{p}$ values $<0.05$ were considered statistically significant. We found no differences between subgroups in any of the psychosocial variables, i.e., health-related quality of life (SF-36), psychological symptoms (BSI), and gender dysphoria (FGI). We therefore tested the influence of these factors without controlling for diagnostic groups.

\section{RESULTS}

\section{Sample Description}

One-hundred and ten adults with DSD participated in the Clinical Evaluation Study. The majority lived as females, and the subgroup DSD-XY-P-M included 11 men with XY-karyotype and partial androgen effects (Table 1). Four participants could not be classified; two of those lived in male gender and two in female gender.

The mean age of the participants was 28.4 years (SD 9.7, range 17-63). Sociodemographic characteristics are shown in Table 1. Active membership in a patient support group was significantly different between subgroups: eight of 17 (47.1\%) DSD-XY-C-F and nine of 32 (28.1\%) DSD-XY$\mathrm{P}-\mathrm{F}$ individuals were members of the respective support groups.

\section{Satisfaction with Care}

Type of Condition. Participants with DSD showed marked differences in general satisfaction with health care as measured with the CSQ (Table 1). Females with DSD-XX (mainly $\mathrm{CAH}$ ) reported the highest satisfaction. Both females and males with DSD-XY conditions and ambiguous phenotype reported moderate satisfaction with care, and women with DSD-XY-C-F (complete androgeninsensitivity syndrome (cAIS) reported lowest satisfaction with care. Differences between DSD-XX and the two DSD$\mathrm{XY}$ conditions living in female gender were significant $(p<$ $0.05)$.

\section{Age Cohort Effects}

Satisfaction with care among the participants was inversely correlated with age $(r=-0.38 p<0.001)$, i.e., older participants were less satisfied than younger respondents. Separate analysis in the four subgroups revealed no significant correlations between satisfaction with care and age within the XX-DSD group, but highly significant correlations within the DSD-XY-C-F group $(r=-0.76, p<0.001)$ DSD-XY-P-F $(r=-0.50, p<0.01)$ and DSD-XY-P-M groups $(r=-0.75, p<0.01)$.

\section{Access to Care}

We asked participants whether they had encountered difficulties with access to specialist care: 31 (28\%) reported such difficulties, and their satisfaction with care was significantly lower (18.5, SD 7.3) compared to those who had not encountered such difficulties (mean 25.4, SD 5.5, $p<0.001)$. We found significantly lower satisfaction with health care in those who reported membership in a patient organization $(p<0.01)$. In the DSD-XY-C-F and DSD-XY-P-F groups, members of support groups reported extremely low satisfaction with care (mean 13.7, SD 4.1 and mean 14.2, SD 6.0) compared to those without membership in such groups (mean 22.1, SD 6.1 and mean 25.1, SD 6.0).

Table 1. Sample Description

\begin{tabular}{|c|c|c|c|c|c|c|}
\hline & $\begin{array}{l}\text { DSD-XX-P-F } \\
N=46\end{array}$ & $\begin{array}{l}\text { DSD-XY-C-F } \\
N=17\end{array}$ & $\begin{array}{l}\text { DSD-XY-P-F } \\
N=32\end{array}$ & $\begin{array}{l}\text { DSD-XY-P-M } \\
N=11\end{array}$ & p Values & $\begin{array}{l}\text { All } \\
N=110\end{array}$ \\
\hline Mean age (SD) & $28.0(7.6)$ & $29.7(8.8)$ & $30.2(12.8)$ & $24.5(9.7)$ & 0.437 & $28.4(9.7)$ \\
\hline Migration background & $3(6.5 \%)$ & $0(0.0 \%)$ & $6(18.8 \%)$ & $2(18.2 \%)$ & 0.099 & $11(10.4 \%)$ \\
\hline Education level & & & & & 0.066 & \\
\hline Low & $11(25.0 \%)$ & $1(6.3 \%)$ & $5(16.7 \%)$ & $2(25.0 \%)$ & - & $22(20.0 \%)$ \\
\hline Medium & $20(45.5 \%)$ & $3(18.8 \%)$ & $8(26.7 \%)$ & $3(37.5 \%)$ & - & $34(30.9 \%)$ \\
\hline High & $9(20.5 \%)$ & $8(50.0 \%)$ & $8(26.7 \%)$ & $3(37.5 \%)$ & - & $29(26.4 \%)$ \\
\hline Very high & $4(9.1 \%)$ & $4(25.0 \%)$ & $9(30.0 \%)$ & $0(0.0 \%)$ & - & $17(15.5 \%)$ \\
\hline Living in current partnership & $14(30.4 \%)$ & $12(70.6 \%)$ & $15(46.9 \%)$ & $2(8.2 \%)$ & 0.010 & $44(40.0 \%)$ \\
\hline Biological children & 9 & 0 & 0 & 0 & 0.007 & \\
\hline Adopted children & $0(0.0 \%)$ & $3(17.6 \%)$ & $3(9.7 \%)$ & $0(0.0 \%)$ & 0.001 & $6(5.5 \%)$ \\
\hline Belonging to a community of faith & $21(45.7 \%)$ & $13(76.5 \%)$ & $15(46.9 \%)$ & $8(72.7 \%)$ & 0.074 & $58(52.7 \%)$ \\
\hline Volunteering in civil or welfare organization & $9(19.6 \%)$ & $5(29.4 \%)$ & $11(34.4 \%)$ & $4(36.4 \%)$ & 0.448 & $32(29.1 \%)$ \\
\hline Member of a condition related self-help group & $1(2.2 \%)$ & $8(47.1 \%)$ & $9(28.1 \%)$ & $1(9.1 \%)$ & $<0.001$ & $20(18.2 \%)$ \\
\hline General satisfaction with care CSQ score & $26.5(4.8)$ & $18.7(6.8)$ & $22.0(7.7)$ & $24.5(3.6)$ & 0.001 & $23.8(6.6)$ \\
\hline (mean, SD, range) & $8-32$ & $10-31$ & $8-32$ & $17-31$ & & $8-32$ \\
\hline
\end{tabular}

CSQ-8 client satisfaction questionnaire, DSD disorders of sex development 


\section{Disclosure and Communication}

We found an inverse correlation among the DSD-XY groups in terms of satisfaction with care and the time required for a diagnosis $(r=0.55, p<0.001)$. In the DSD$\mathrm{XX}$ group there was no such association. The circumstances and the person disclosing the diagnosis had no impact on the level of satisfaction with care, but the extent to which participants understood the information had a significant impact. Participants who reported not understanding the information at the time of disclosure reported lower scores of satisfaction with care (mean 21.3, SD 7.5) compared to those who had partly (mean 22.6, SD 7.4) or fully (mean 23.2, SD 7.3) understood the information.

\section{Surgery}

Ninety-four participants had undergone surgical procedures related to their condition. The overall rating of the results of surgery in terms of overall appearance and sexual functioning did not differ among the four groups. The results related to overall appearance and sexual functioning had a significant impact on overall satisfaction with care (Table 2).

Men with XY-DSD rated the results relating to urinary functioning significantly lower compared to the other groups (six of 11 of XY-DSD-P-M individuals rated urinary function after surgery as unsatisfactory or bad versus two of the 29 XY-DSD-P-F individuals and none of the two other groups). This group had undergone $>2$ operations significantly more often $(39.6 \%)$ than women with XY-
DSD-P conditions $(25.0 \%)$ and the other two groups (< $10 \%)$.

Seventy-five participants had undergone plastic constructive surgery, defined as procedures aimed at masculinization (i.e., testes implantation, penis prosthesis implantation, testis fixation procedures, augmentation of the penis, mastectomy, vaginectomy, or hysterectomy in males) or feminization (i.e., clitoris reduction, construction of the vagina, construction of the labia, and resection of the ductus deferens). Among the women who underwent plastic constructive surgery, XX-DSD-F women reported highest (24.3, SD 3.9) and women with XY-DSD-P lowest levels of satisfaction with care (22.3, SD 7.1; differences $p<0.05)$. Across all groups, those who had undergone plastic constructive surgery reported higher levels of satisfaction with care compared to participants who had not undergone such procedures (mean 25.1 SD 5.6 vs. 20.9, SD 7.8; $p<0.05)$.

\section{Psychological Support}

Twelve participants $(11 \%)$ reported having been offered and using psychological counseling (including psychotherapy) in adulthood; others $(31 ; 28 \%)$ reported that they had been offered such services but that they had no need for them. The majority reported that they had never been offered such services: among them were 30 (27 \%) participants who reported unmet needs (data shown in Table 2). The latter group reported the lowest satisfaction with care. Comparison of the group who had received services and the three groups who had not, did not reveal

Table 2. General Satisfaction with Care in Relationship with the Rating of Surgical Procedures and the Receipt of Psychological Counseling

\begin{tabular}{|c|c|c|c|}
\hline & $\mathbf{N}$ & Mean (SD) of CSQ-8 & $\overline{\text { p Values }}$ \\
\hline \multicolumn{4}{|l|}{ How do you rate the overall results of your surgical procedures in relationship to appearance? } \\
\hline Good & 56 & $26.1(5.7)$ & \multirow[t]{4}{*}{$<0.001$} \\
\hline Average & 20 & $22.4(6.4)$ & \\
\hline Unsatisfactory & 11 & $21.1(6.6)$ & \\
\hline Bad & 5 & $11.6(3.8)$ & \\
\hline \multicolumn{4}{|l|}{ How do you rate the overall results of your surgical procedures in relationship to sexual functioning? } \\
\hline Good & 52 & $25.3(6.0)$ & \multirow[t]{4}{*}{0.008} \\
\hline Average & 17 & $23.8(7.3)$ & \\
\hline Unsatisfactory & 7 & $19.8(5.4)$ & \\
\hline $\mathrm{Bad}$ & 9 & $18.3(7.4)$ & \\
\hline \multicolumn{4}{|l|}{ How do you rate the overall results of your surgical procedures in relationship to urinary functioning? } \\
\hline Good & $77^{\circ}$ & $24.6(6.5)$ & \multirow{4}{*}{0.198} \\
\hline Average & 6 & $17.5(8.4)$ & \\
\hline Unsatisfactory & 5 & $24.0(1.9)$ & \\
\hline Bad & 1 & - & \\
\hline \multicolumn{4}{|l|}{ Psychological counseling } \\
\hline I have been offered and used psychological counseling (receipt of services) & $12(11 \%)$ & $22.0(6.5)$ & \multirow[t]{4}{*}{$<0.001$} \\
\hline I been offered counselling, but did not need such services & $31(28 \%)$ & $26.8(5.2)$ & \\
\hline I have not been offered psychological counselling, but would not have needed such services & $34(31 \%)$ & $24.8(5.5)$ & \\
\hline $\begin{array}{l}\text { I have never been offered psychological counselling, but would have needed } \\
\text { such services (unmet needs) }\end{array}$ & $30(27 \%)$ & $19.3(7.4)$ & \\
\hline
\end{tabular}

$n=2$ missing data for surgical procedures, $n=3$ for psychological counseling

CSQ-8 client satisfaction questionnaire 
significant differences. The only highly significant differences $(p<0.01)$ were between those who had not received services congruent with their wishes (regardless whether they had been offered services or not) and those who had unmet needs.

\section{Personal Factors and Well-Being}

Rates of gender dysphoria did not differ among the four subgroups (for full results for the FGI, see Jürgensen et al. ${ }^{19}$ ). We found a highly significant inverse correlation between the transgender scale indicating gender dysphoria and satisfaction with care in the full sample, i.e., the more gender dysphoria individuals experienced, the less satisfied they were $(r=0.44 ; p<0.001)$.

One-fifth of all participants reported symptoms in a clinically relevant range ( $t$-score $\geq 63$ ) of the BSI. In those with a higher burden of symptoms, satisfaction with health care was significantly lower compared to those with fewer psychological symptoms (mean 24.6, SD 6.4 vs. 19.3, SD 7.0). There were no differences between diagnostic subgroups concerning this relationship.

We found mild inverse correlations between psychological symptoms and satisfaction with care in the dimensions of somatization, insecurity, phobia, and psychotic symptoms (correlation coefficients ranging between $r=0.18$ to $0.22, p<0.05)$. Satisfaction with health care was also correlated with health-related quality of life (SF-36) in the areas of vitality $(r=0.30, p<0.01)$, social role functioning ( $r=0.36, p<0.001)$, emotional role functioning $(r=0.42, p<$ $0.001)$, and emotional well-being $(r=0.25, p<0.05)$.

\section{DISCUSSION}

All DSD groups reported lower satisfaction with care (mean 23.8, SD 6.6) compared to adult patients with other serious chronic conditions in rehabilitation services (mean 27.00, SD 4.01, $n=53.177) .{ }^{20}$ The group most satisfied among the four condition groups was women with XX-DSD, predominantly individuals with $\mathrm{CAH}$. We were not surprised that patients treated in clinics serving larger cohorts with an established and definite diagnosis and available treatments, such as $\mathrm{CAH}$, are more satisfied. Within this group, neither age cohort nor time to diagnosis had an effect on satisfaction with care, indicating that organization and processes of care for CAH have been fairly stable over the last decades. The requirement for lifelong daily medication results in better patient education and continuity of care, features related to higher satisfaction with care. We also speculate that individuals with XX-DSD are well educated about their condition early on, and children and families adapt to the daily management of a chronic condition. In western countries, girls with $\mathrm{CAH}$ are raised in the female gender despite frequent excessive virilization, and thus there is no discrepancy between karyotype and gender. However, in $\mathrm{CAH}$ there may persist some reluctance to address issues of psychosexual development; long-term outcomes in psychosexual health have been shown to be unsatisfactory in this patient group, ${ }^{21,22}$ which may contribute to some low satisfaction scores in our sample.

In DSD-XY, the situation is different indeed: for many patients the time to diagnosis is extremely long and in some cases, no definite molecular diagnosis is possible. Most of these individuals have no immediate medical care needs, and the focus of concern is psychosexual development, reproduction, and gender assignment. ${ }^{23,24}$ Males with XYDSD conditions often undergo multiple surgical procedures. However, this small group reported higher overall satisfaction with care compared to their female counterparts. Matching the karyotype with the sex of rearing may help both patients and physicians feel more comfortable addressing the consequences of undervirilization. In females with complete forms of XY-DSD (and unambiguous genitalia and normal breast development), the impact of secrecy and poor communication about the condition contributes to the psychological burden. ${ }^{25}$ Because many adults with XYDSD were denied full disclosure when they were children, the feeling of being different without understanding the reasons and lack of family support may contribute to their psychological burden. Care is generally fragmented in this group, and patients or parents may have difficulties finding a specialist and/or may consult many different specialists from various fields, often with conflicting recommendations. Primary care physicians may feel unprepared to assume case management.

We found that longer time to diagnosis, suboptimal outcome of surgical procedures, and lack of access to specialist care were all associated with low satisfaction with care (see also Köhler et al. ${ }^{26}$ ). Individuals who had plastic constructive surgery reported higher satisfaction with care than those who had no such procedures, and access to procedures aimed at better appearance and better functioning may be important for increasing satisfaction with care. Because the rating of the outcome of surgical procedures was significantly associated with general satisfaction with care, we recommend that surgical centers report to clinical registries on patients, procedures, and outcomes at regular follow-up intervals to help improve the quality of care.

We have shown that patients without access to psychosocial care are less satisfied with their health care. The burden and effect of these conditions on daily life, aspirations, and adaptation to the situation may cause impairments in physical, emotional, and social wellbeing. ${ }^{27,28}$ Whether participants received psychological counseling did not affect their satisfaction with care, but unmet needs did. Psychosocial interventions should be 
limited to those who need and want such services. ${ }^{29}$ Approximately one-third of our participants had unmet needs in this area, and their level of satisfaction was lowest. We recommend that both primary care providers as well as medical specialists be vigilant for psychological symptoms during the life course of individuals with DSD, and ensure that counseling or psychotherapy is available. Because DSD is a complex condition affecting deeply rooted issues such as gender identity, sexuality, partnership, life aspirations, and family relations, counselors and therapists should be well informed about the condition and hopefully work as member of a multidisciplinary team. ${ }^{30}$

All measures of general well-being were highly correlated with satisfaction with care across the groups. Higher satisfaction with care was associated with higher levels of health-related quality of life in the dimensions of emotional and social well-being; lower levels of psychological symptoms in the dimensions of somatization, insecurity, phobia, and psychotic symptoms; and lower risk for gender dysphoria. As this is a cross-sectional study, we do not fully understand the trajectory of this association. The association may be causal in both directions: (1) well people with high levels of social well-being may be more likely to find access to good care, and (2) people with unsatisfactory health care and lack of access to psychosocial care experience are burdened by the condition and report lower quality of life and more psychological symptoms.

We conducted a naturalistic observational study based on the fact that scientific knowledge has not been sufficient to generate a-priori hypotheses. The inclusion of different etiological groups is important to generate hypotheses regarding the relevance of genetic, hormonal, psychological, and social influence on the patients' physical, emotional and social well-being. Typical treatment sequences and decisions for special interventions are explored as potential factors influencing satisfaction with care. A limitation of the study is the lack of population-based representativeness due to recruitment bias. We noted very different levels of active participation in patient support groups, with especially high rates in the female XY-DSD groups. We speculate that membership in a patient organization that assumes a strong advocacy role may be associated with past dissatisfaction and poor treatment experiences. Unlike other studies, our recruitment was largely through specialized centers and clinics, but the support of certain patient organizations may have resulted in a selection bias. ${ }^{31}$ Another limitation is the cross-sectional nature of the study, which does not allow inference to causal relationships. Long-term, prospective outcome studies are necessary to further develop guidelines for individuals with DSD in all age groups. Using a lifetime perspective, long-term outcome studies must begin in childhood and must also address parents' needs. As in other chronic conditions beginning in childhood, continuity of care and communication between professionals and inter- agency collaboration improve the quality of care and outcomes. ${ }^{32}$ Further, transition to adult health care services requires the early anticipation of adolescents' needs. ${ }^{33}$

\section{CONCLUSIONS}

Results from this study support the following recommendations: (1) individuals with DSD should have access to experienced centers for rare conditions with access to mental health services as part of interdisciplinary care; (2) long-term follow-up should include measures of satisfaction with care and subjective psychosexual, emotional, and social well-being; and (3) the methodology of observational treatment studies should be further developed to ensure full participation and avoid recruitment bias.

Acknowledgements: We are very grateful to all study participants; without their openness and commitment to talk about very intimate aspects of their lives, the study would not have been possible. We would like to thank Eva Hampel, Esther Müller-Godeffroy, Heino Meyer-Bahlburg, and Hertha Richter-Appelt for methodological support and the interviewers in Germany, Austria and Switzerland. Many thanks also to the following: Wiebke Naujoks and Pia Widenka for collecting control data, Sabine Lorenz for her organizational support during the entire study, and Grit Hambruch for her data entry work. Special thanks go to all cooperating physicians in the DSD Network Working group who were willing to help with recruitment and clinical data. The study was supported by the European Scientific Advisory Board: Silvano Bertelloni; Peggy T. Cohen-Kettenis, Ieuan A. Hughes, Claire Nihoul-Fékété, Martin Ritzén, Justine M. Schober. The study was funded by the German Ministry of Education and Science (FKZ 01GM0311).

Conflict of Interest: The authors declare that they do not have a conflict of interest.

Corresponding Author: Ute Thyen, MD; Hospital for Children and Adolescents, University Lübeck, Lübeck, Germany (e-mail: Ute.thyen@uksh.de).

\section{REFERENCES}

1. Hughes IA, Houk C, Ahmed SF, Lee PA, Lawson Wilkins Pediatric Endocrine Society/European Society for Paediatric Endocrinology Consensus Group. Consensus statement on management of intersex disorders. Arch Dis Child. 2006;91:554-63.

2. Joint LWPES/ESPE CAH Working Group. Consensus statement on 21hydroxylase deficiency from The Lawson Wilkins Pediatric Endocrine Society and The European Society for Paediatric Endocrinology. J Clin Endocrinol Metab. 2002;87:4048-53.

3. Speiser PW, Azziz R, Baskin LS, et al. Congenital adrenal hyperplasia due to steroid 21-hydroxylase deficiency: an Endocrine Society Clinical Practice Guideline. J Clin Endocrinol Metab. 2010;95:4133-60.

4. Thyen U, Lanz K, Holterhus PM, Hiort O. Epidemiology and initial management of ambiguous genitalia at birth in Germany. Horm Res. 2006;66:195-203.

5. Thyen U, Richter-Appelt H, Wiesemann C, Holterhus PM, Hiort O. Deciding on gender in children with intersex conditions: considerations and controversies. Treat Endocrinol. 2005;4:1-8. 
6. Money J, Hampson JG, Hampson JL. Hermaphroditism: recommendations concerning assignment of sex, change of sex, and psychologic management. Bull Johns Hopkins Hosp. 1955;97:284-300.

7. Money J, Ehrhardt AA. Man \& Woman. Boy \& Girl: differentiation and dimorphism of gender identity from conception to maturity. Baltimore: Johns Hopkins University Press; 1972.

8. Austin J, Tamar-Mattis A, Mazur T, Henwood MJ, Rossi WC. Disorders of sex development-when and how to tell the patient. Pediatr Endocrinol Rev. 2011;8:213-7.

9. Diamond M, Sigmundson HK. Management of intersexuality. Guidelines for dealing with persons with ambiguous genitalia. Arch Pediatr Adolesc Med. 1997;151:1046-50.

10. Dreger AD. "Ambiguous sex"-or ambivalent medicine? Ethical issues in the treatment of intersexuality. Hastings Cent Rep. 1998;28:24-35.

11. Meyer-Bahlburg HM. Sex steroids and variants of gender identity. Endocrinol Metab Clin N Am. 2013;42:435-52.

12. Wiesemann C, Ude-Koeller S, Sinnecker GH, Thyen U. Ethical principles and recommendations for the medical management of differences of sex development (DSD)/intersex in children and adolescents. Eur J Pediatr. 2010;169:671-9.

13. World Health Organization. Work book 6: client satisfaction evaluations. Available at: http://www.emcdda.europa.eu/attachements.cfm/ att_5868_EN_6_client_satisfaction_evaluations.pdf. Accessed May 2014

14. Lux A, Kropf S, Kleinemeier E, Jürgensen M, Thyen U, DSD Network Working Group. Clinical evaluation study of the German network of disorders of sex development (DSD)/intersexuality: study design, description of the study population, and data quality. BMC Public Health. 2009;9:110. Open access at http://www.biomedcentral.com/1471 2458/9/110, accessed May, 2014.

15. Schmidt J, Wittmann WW. ZUF-8 Fragebogen zur Messung der Patientenzufriedenheit. In: Brähler J, Schuhmacher J, Strauß B (eds.) Diagnostische Verfahren in der Psychotherapie. Göttingen:Hogrefe; 2002:392-6.

16. Bullinger M, Kirchberger I. Der SF-36 Fragebogen zum Gesundheitszustand - Handbuch für die deutschsprachige Fragebogen-Version. Göttingen: Hogrefe; 1998.

17. Franke GH. Brief Symptom Inventory. Deutsche Version. Manual:Göttingen, Beltz Test; 2000.

18. Richter Appelt H, Eckloff T. Questionnaire of Gender Identity. (German Title: Fragebogen zur Geschlechtsidentität). Unpublished manuscript, University of Hamburg.

19. Jürgensen M, Kleinemeier E, Lux A, et al. Psychosexual development in adolescents and adults with disorder of sex development (DSD) results from the German Clinical Evaluation Study. J Sex Med. 2013;10:2701-14.

20. Kriz D, Nübling $\mathbf{R}$, Steffanowski A, Rieger J, Schmidt J. Patientenzufriedenheit: Psychometrische Reanalyse des ZUF-8.
Berlin:Deutsche Rentenversicherung Bund. DRV-Schriften, 2008;77:84-5.

21. Nordenskjöld A, Holmdahl G, Frisen L, et al. Type of mutation and surgical procedure affect long-term quality of life for women with congenital adrenal hyperplasia. J Clin Endocrinol Metab. 2008;93:3806.

22. Nordenström A. Adult women with 21-hydroxylase deficient congenital adrenal hyperplasia, surgical and psychological aspects. Curr Opin Pediatr. 2010;23:436-42.

23. Massanyi EZ, Dicarlo HN, Migeon CJ, Gearhart JP. Review and management of 46, XY disorders of sex development. J Pediatr Urol. 2013;9:368-79.

24. Streuli JC, Köhler B, Werner-Rosen K, Mitchell C. DSD and professionalism from a multilateral view: supplementing the consensus statement on the basis of a qualitative survey. Adv Urol. 2012. doi: $10.1155 / 2012 / 185787$.

25. Kleinemeier E, Jürgensen M, Lux A, Widenka PM, Thyen U, DSD Network Working Group. Psychological adjustment and sexual development of adolescents with disorders of sex development. J Adolesc Health. 2010;47:463-72.

26. Köhler B, Kleinemeier E, Lux A, et al. Satisfaction with genital surgery and sexual life of adults with XY disorders of sex development - results from the German Clinical Evaluation Study. J Clin Endocrinol Metab. 2012;97:577-88.

27. Nermoen I, Husebye ES, Svartberg J, Løvås K. Subjective health status in men and women with congenital adrenal hyperplasia: a population-based survey in Norway. Eur J Endocrinol. 2010;163:453-9.

28. Wisniewski AB, Mazur T. $46, \mathrm{XY}$ DSD with female or ambiguous external genitalia at birth due to androgen insensitivity syndrome, 5alphareductase-2 deficiency, or 17beta-hydroxysteroid dehydrogenase deficiency: a review of quality of life outcomes. Int $\mathrm{J}$ Pediatr Endocrinol. 2009;567430.

29. Sandberg DE, Gardner M, Cohen-Kettenis PT. Psychological aspects of the treatment of patients with disorders of sex development. Semin Reprod Med. 2012;30:443-52.

30. Palmer BW, Wisniewski AB, Schaeffer TL, et al. A model of delivering multi-disciplinary care to people with $46 \mathrm{XY}$ DSD. J Pediatr Urol. 2012;8:7-16.

31. German Ethics Council. Intersexuality. Available at: http:// www.ethikrat.org/files/opinion-intersexuality.pdf. Accessed May, 2014.

32. Palfrey JS, Sofis LA, Davidson EJ, et al. The Pediatric Alliance for Coordinated Care: evaluation of a medical home model. Pediatrics. 2004;113(5 Suppl):1507-16.

33. Hullmann SE, Chalmers LJ, Wisniewski AB. Transition from pediatric to adult care for adolescents and young adults with a disorder of sex development. J Pediatr Adolesc Gynecol. 2012;25:155-7. 